\title{
Extended coherence lifetimes in microcavities under angle-resonant pumping conditions
}

Østergaard, John Erland; Birkedal, Dan; Mizeikis, V.; Jensen, Jacob Riis; Hvam, Jørn Märcher

Published in:

Technical Digest. Quantum Electronics and Laser Science Conference.

Link to article, DOI:

10.1109/QELS.2001.962147

Publication date:

2001

Document Version

Publisher's PDF, also known as Version of record

Link back to DTU Orbit

Citation (APA):

Østergaard, J. E., Birkedal, D., Mizeikis, V., Jensen, J. R., \& Hvam, J. M. (2001). Extended coherence lifetimes in microcavities under angle-resonant pumping conditions. In Technical Digest. Quantum Electronics and Laser Science Conference. (pp. 232-233). Opt. Soc. America. https://doi.org/10.1109/QELS.2001.962147

\section{General rights}

Copyright and moral rights for the publications made accessible in the public portal are retained by the authors and/or other copyright owners and it is a condition of accessing publications that users recognise and abide by the legal requirements associated with these rights.

- Users may download and print one copy of any publication from the public portal for the purpose of private study or research.

- You may not further distribute the material or use it for any profit-making activity or commercial gain

- You may freely distribute the URL identifying the publication in the public portal 


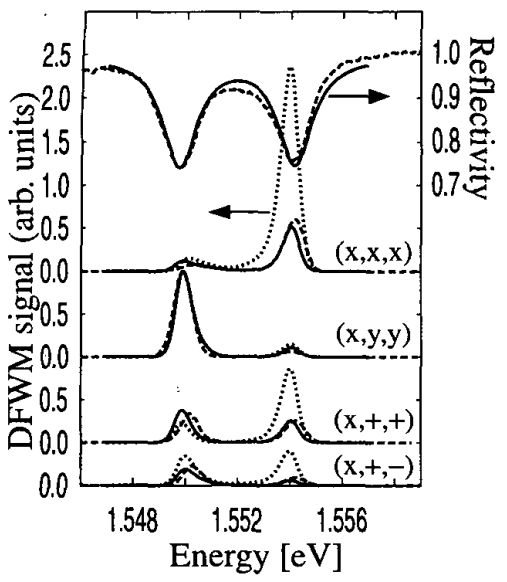

QThL3 Fig. 1. Frequency-degenerate fourwave-mixing signals from a quantum-well microcavity. Long-dashed: experiment (from ${ }^{2}$ ); solid: theory (exact exciton-exciton scattering amplitude); short-dashed: theory (second Born). The abscissa is the beam frequency (for both pump and probe). The beam polarizations are designated in the order (pump, probe, signal). The cavity resonance is tuned to the exciton resonance $(1.552 \mathrm{eV})$. The linear reflectivity spectrum is also shown.

2. M. Gonokami, S. Inoue, H. Suzuura, M. Shirane, and R. Shimano, Phys. Rev. Lett. 79, 1341 (1997).

3. V.M. Axt and A. Stahl, Z. Phys. B 93, 195 (1994).

4. W. Schäfer, D. Kim, J. Shah, T. Damen, J. Cunningham, K. Goossen, L. Pfeiffer, and K. Köhler, Phys. Rev. B 53, 16429 (1996).

5. P. Kner, S. Bar-Ad, M.V. Marquezini, D.S. Chemla, and W. Schäfer, Phys. Rev. Lett. 78, 1319 (1997).

6. R. Binder, I. Rumyantsev, N.H. Kwong, and R. Takayama, Phys. Stat. Sol. (b) 221, 169 (2000).

Quantum Correlations in a Semiconductor Microcavity

P. Brick, C. Ell, M. Hübner, E.S. Lee, O. Lyngnes, J.P. Prineas, G. Khitrova, H.M. Gibbs, Optical

Sciences Center, University of Arizona, Tucson, AZ 85721; Email: brick@optics.arizona.edu

M. Kira, F. Jahnke, S.W. Koch, Department of Physics and Material Sciences Center, Philipps University, Renthof 5, D-35032 Marburg, Germany

D.G. Deppe and D.L. Huffaker, Microelectronics Research Center, University of Texas at Austin, Austin, TX 78758

The nonlinear optical response of semiconductor microcavities in the nonperturbative regime has been the subject of many recent experiments. Contrary to clear demonstrations of the quantized nature of the vacuum-field Rabi splitting of a single atom, most of the experiments on semiconductor microcavities could be explained in terms of a classical light field. Only recently, quan- tized light field effects have been reported in a coherent control experiment ${ }^{1}$ and theoretically investigated in secondary emission. ${ }^{2}$

Here, results are presented that display a pronounced third transmission maximum lying energetically between the two normal modes. The third peak can be observed in resonant femtosecond single-beam as well as picosecond pumpprobe experiments under various excitation conditions. This finding is strikingly different from the, in general, double-peaked transmission in the nonperturbative regime of a semiconductor microcavity. It is even more in contrast to the case of nonresonant excitation, where at most two peaks can be observed. In the latter case, the two normal-mode peaks broaden with increasing

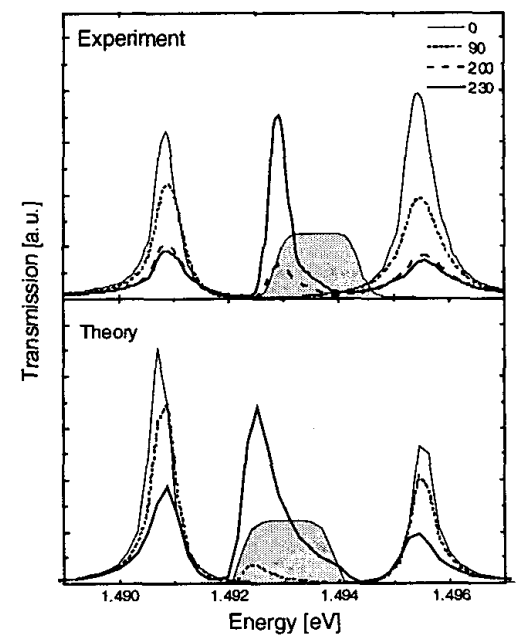

QThL4 Fig. 1. Transmission of the microcavity for various pump pulse intensities $\left.[\mathrm{n}] / \mathrm{cm}^{2}\right]$ for co-circular polarization and zero pumpprobe delay. The shaded regions indicate the pump spectrum.

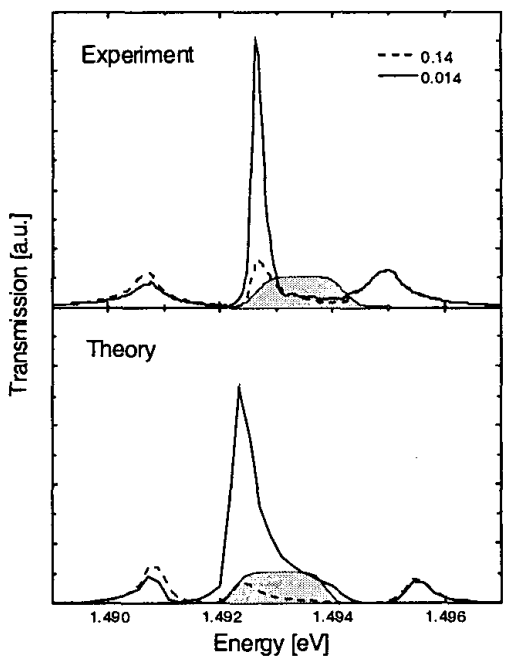

QThL4 Fig. 2. Dependence of microcavity transmission on probe intensity $\left.[\mathrm{n}] / \mathrm{cm}^{2}\right]$ at constant pump intensity of $330 \mathrm{~nJ} / \mathrm{cm}^{2}$ for co-circular polarization and zero pump-probe delay. The shaded regions indicate the pump spectrum. pump-intensity and finally collapse into a single peak indicating the onset of the weak coupling regime.

The origin of this distinct effect is analyzed using a fully quantum mechanical approach that combines the semiconductor luminescence equations and the semiconductor Bloch equations formulated for a quantized light field. ${ }^{2}$ In particular, the equation of motion for the interband polarization is modified if the quantization of the light field is taken into account. These quantum corrections include contributions due to the emission into guided modes. Consequently, the polarization in the normal direction, thus the transmission, is influenced by intraband correlations with photons propagating in all other directions.

Figure 1 shows the calculated and measured transmission spectra under the influence of a resonant pump beam. Both theory and experiment reveal that the third peak follows the low energy side of the pump pulse and shifts little with pump intensity. Here, the quantum corrections create in the probe spectrum a weak coupling component that has similar characteristics as the pump pulse.

Even more intriguing is the dependence of the transmission spectrum on the probe intensity itself at constant pump intensity; see Fig. 2. Because the quantum corrections are mainly created by the pump pulse, the relative effect on the probe dynamics increases with decreasing probe intensities. In good agreement with the theoretical prediction the experimentally observed third peak becomes more pronounced for smaller probe intensities. ${ }^{3}$

1. Y.-S. Lee, T.B. Norris, M. Kira, F. Jahnke, S.W. Koch, G. Khitrova, and H.M. Gibbs, "Quantum Correlations and Intraband Coherences in Semiconductor Cavity QED", Phys. Rev. Lett. 83, 5338 (1999).

2. M. Kira, F. Jahnke, and S.W. Koch, "Quantum Theory of Secondary Emission in Optically Excited Semiconductor Quantum Wells", Phys. Rev. Lett. 82, 3544 (1999).

3. C. Ell, P. Brick, M. Hübner, E.S. Lee, O. Lyngnes, J.P. Prineas, G. Khitrova, H.M. Gibbs, M. Kira, F. Jahnke, S.W. Koch, D.G. Deppe, and D.L. Huffaker "Quantum Correlations in the Nonperturbative Regime of Semiconductor Microcavities", accepted for publication in Phys. Rev. Lett.

QThL5

3:30 pm

Extended coherence lifetimes in microcavitles under angle-resonant pumplng condltions

John Erland, Dan Birkedal, Vygantas Mizeikis, Jacob R. Jensen, and Jørn M. Hvam, Research Center COM, Technical University of Denmark, Oersteds Plads 349, DK-2800 Kgs. Lyngby, Denmark; Email: jeo@com.dtu.dk

Strong laser-like emission from polaritons in microcavities has been observed and attributed to bosonic enhancement in the emission process. ${ }^{I-3}$ This relies on the bosonic nature of low-density polaritons stimulating the scattering processes by the final-state polariton population. Strong enhancements of the scattering processes have been observed as a result of up to several 100 polaritons per final $\mathrm{k}$-state under angle-resonant 
pumping conditions. ${ }^{4,5}$ Energy and wave vector are conserved in the angle-resonant process by scattering of two $k_{\|}=k_{\|, \text {res }}$ polaritons into $2 k_{\|, \text {,res }}$ and $k_{y} \sim 0$ states, respectively, the latter resulting in the strong secondary emission that is studied in this work.

In the $5 \mathrm{~K}$ experiments, the time-resolved secondary emission, from a microcavity with a $10 \mathrm{~nm}$ single quantum well, is selected around $\mathrm{k}_{\mathrm{f}} \sim 0$ (Fig. $1(\mathrm{a})$ ) after angle-resonant pulsed excitation (see Fig. 1(b)). ${ }^{3}$ The angle-resonance illustrated in Fig. 1(b) is achieved at zero detuning in this work. At the lowest density, a long single exponential decay is observed, that evolves into a much faster excitation-dependent emission transient for higher densities. The turnover corresponds to a final-state density with more than 1 polariton per k-state resulting in the faster emission dynamics due to the final-state stimulation.

In the work presented here we are interested in probing the coherences involved in the bosonic enhancement of the polariton-polariton scattering process. As illustrated in Fig. 1(b) we probe the coherences in the final-state with a four-wave mixing (FWM) experiment without or with simultaneous pumping at the angle-resonance as in Fig. 1(a). From the experiments without pumping (dashed curve in Fig. 2), a FWM decay time of $9 \mathrm{ps}\left(\mathrm{T}_{2}=18 \mathrm{ps}\right)$ results in a line width of $75 \mu \mathrm{eV}$ consistent with spectral analysis of the secondary emission. Measuring the FWM, as a function of time-delay between the two pulses, probes the decay of the polarization induced by the first pulse. Thus, any coherent process that adds coherently to that polarization will directly be reflected in the time-delay dependence in the FWM experiment. In our preliminary FWM data with the angle-resonant pump present we indeed find strongly modified time-delay dependences as a result of the final-state stimulated polaritonpolariton scattering. As seen in Fig. 2 for moderate intensities $(0.2 \mathrm{~mW}$ and $0.4 \mathrm{~mW}$ as indicated by the arrow) and longer delays, we obtain a balance between the loss of coherence, due to the polariton lifetime in the cavity, and the stimulated polariton-polariton scattering that maintains the coherence for longer times. Thus, the time duration of the polarization induced by the first pulse is extended, however, with a complicated tran-

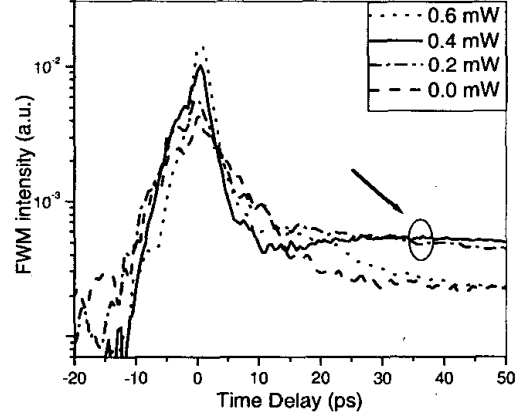

QThL5 Fig. 2. FWM-intensity versus timedelay with simultaneous non-delayed pumping at the angle-resonant conditions sketched in Fig. 1(b). Compared to no pumping, an extended coherence lifetime is observed for longer delays as indicated by the arrow with the average intensities shown in the inset.

sient behaviour. This is furthermore a direct measurement of the coherent nature of the finalstate stimulated scattering process. For higher intensities, the extension is limited because of the stronger stimulated dynamics just as in the timeresolved secondary emission presented in Fig. 1 (a).

These preliminary FWM data thus demonstrates that the stimulated polariton-polariton scattering in microcavities build up a coherence in the final-state or gives the possibility of extending the coherence lifetime of an induced polarization. Further studies will include dependences on e.g. temperature and detuning and will in greater detail explore the possibilities with these coherence-maintaining processes in semiconductor microcavities.

\section{References}

1. P. Snellart and J. Bloch, 'Nonlinear Emission of Microcavity Polaritons in the Low Density Regime', Phys. Rev. Lett. 82, 1233 (1999).

2. P.G. Savvidis, J.J. Baumberg, R.M. Stevenson, M.S. Skolnick, D.M. Whittaker, and I.S. Roberts, 'Angle-resonant stimulated polari-

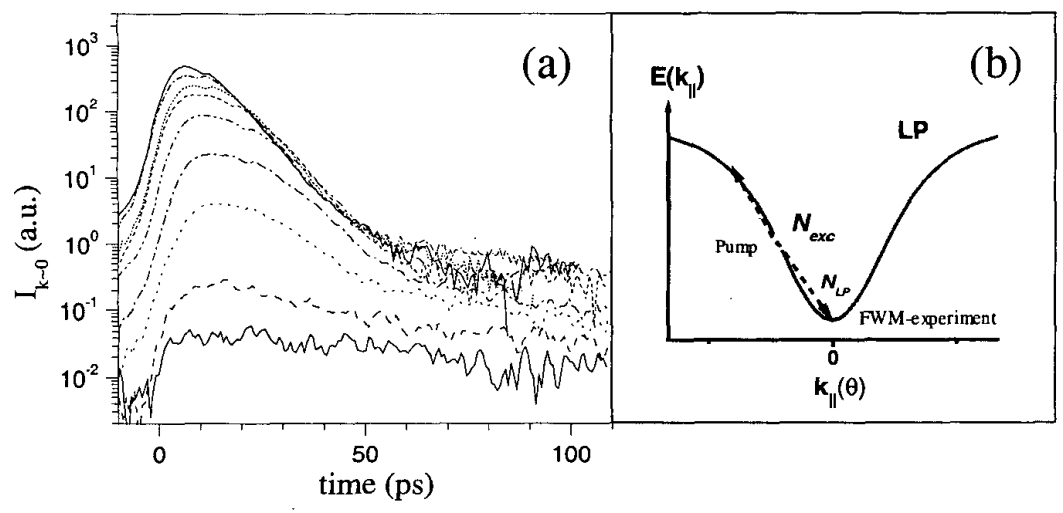

QThL5 Fig. 1. a) Time-resolved secondary emission detected at a zero in-plane wave-vector showing a fast emission transient after angle-resonant single pump pulse excitation for polariton-polariton scattering shown in b). Also indicated is the configuration for the FWM experiment (see results in Fig. 2) with two incident laser pulses at small $\mathrm{k}_{\text {f. }}$. A clear excitation density dependence of the emission dynamics in a) from $5 \cdot 10^{8} \mathrm{~cm}^{-2}$ to $2 \cdot 10^{10} \mathrm{~cm}^{-2}$ is observed. ton amplifier', Phys. Rev. Lett. 84, 1547 (2000).

3. J. Erland, V. Mizeikis, W. Langbein, J.R. Jensen, N.A. Mortensen, and J.M. Hvam, 'Seeding of polariton stimulation in a homogeneously broadened microcavity', phys. stat. sol. (b) 221, 115 (2000).

4. J. Erland, V. Mizeikis, W. Langbein, J.R. Jensen, and J.M. Hvam, 'Stimulated secondary emission from semiconductor microcavities', submitted to Phys. Rev. Lett., June 2000.

5. R.M. Stevenson, et al., 'Continuous wave observation of massive polariton redistribution by stimulated scattering in semiconductor microcavities', Phys. Rev. Lett. 85, 3680 (2000).

Biexciton dephasing in a semiconductor microcavity

P. Borri, W. Langbein, and U. Woggon, Universität Dortmund, Otto-Hahn Str. 4, 44221 Dortmund, Germany; Email: borri@fred.physik.uni-dortmund.de

J.R. Jensen, and J.M. Hvam, Research Center COM, The Technical University of Denmark DK-2800 Kgs. Lyngby, Denmark

The experimental observation of biexcitons in microcavities has been addressed recently. ${ }^{1,2} \mathrm{~A}$ well-resolved polariton-biexciton transition was observed in a high-quality GaAs single quantum well (OW) $\lambda$-microcavity of $25 \mathrm{~nm}$ well width using a pump-probe experiment. ${ }^{2}$ In this microcavity the heavy-hole $(\mathrm{HH})$ vacuum Rabi splitting is $3.6 \mathrm{meV}$, more than three times larger than the biexciton binding energy in the bare QW (1.1 $\mathrm{meV})$. Due to the narrow linewidth of the polariton resonances, a well-resolved pump-induced optical absorption associated with biexcitons was observed. ${ }^{2}$ In this work we investigate the coherent properties of the biexciton in the same structure using a two-beam four-wave mixing (FWM) experiment at $10 \mathrm{~K}$. The exciting pulses were propagating along $\mathbf{k}_{1}$ and $\mathbf{k}_{2}$ directions at small incident angles $\left(\sim 1^{\circ}\right)$, with the pulse along $k_{1}$ leading at positive delay times $\tau_{12}$. In Fig. 1 the

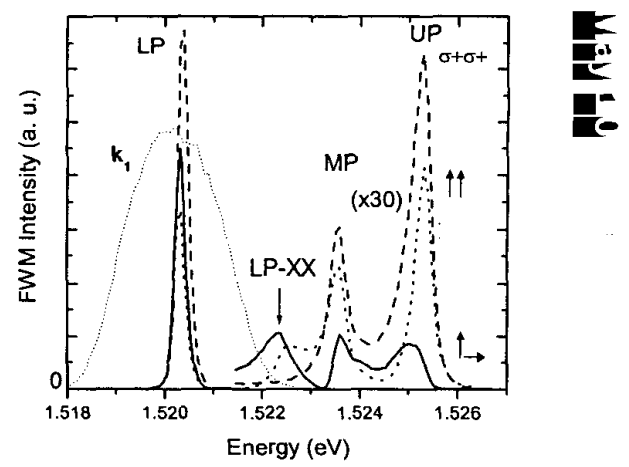

QThL6 Fig. 1. Spectrally resolved four-wave mixing for co-circular $(\sigma+\sigma+$, dashed line) colinear $(\uparrow \uparrow$, bold dotted line) and cross-linear ( $\rightarrow \uparrow$, bold line) polarizations. The spectrum of the $k_{1}$ pulse is also shown (dotted line). 